Kragujevac Journal of Mathematics

Volume 45(6) (2021), Pages 977-994.

\title{
PSEUDO COMMUTATIVE DOUBLE BASIC ALGEBRAS
}

\author{
SHOKOOFEH GHORBANI ${ }^{1}$
}

\begin{abstract}
In this paper, we study the concept of pseudo commutative double basic algebras and investigate some related results. We prove that there are relations among pseudo commutative double basic algebras and other logical algebras such as pseudo hoops, pseudo BCK-algebras and double MV-algebras. We obtain a close relation between pseudo commutative double basic algebras and pseudo residuted l-groupoids. Then we investigate the properties of the boolean center of pseudo commutative double basic algebras and we use the boolean elements to define and study algebras on subintervals of pseudo commutative double basic algebras.
\end{abstract}

\section{INTRODUCTION}

C. C. Chang introduced the concept of MV-algebra in 1958 [16] to prove the completeness theorem of infinite valued Łukasiewicz propositional calculus. Basic algebras were introduced by I. Chajda et al. as a generalization of MV-algebras and orthomodular lattices, see $[8,10]$ and $[11]$. They are very useful in non-classical logics including the logic of quantum mechanics. Every basic algebra is in fact a bounded lattice with the so-called section antitone involutions.

I. Chajda introduced the concept of double basic algebra in 2009 [12] as a generalization of basic algebra. Double basic algebras are determined by two binary, two unary and a nullary operation satisfying similar axioms to those defining basic algebras. Hence the class of these algebras forms a variety. He proved that there exists a one-to-one correspondence between the variety of double basic algebras and the class of lattices with section antitone bijections. Also, he obtained the relation between double MV-algebras and double basic algebras.

Key words and phrases. Pseudo commutative double basic algebra, double MV-algebra, pseudo residuted l-groupoid, boolean element.

2010 Mathematics Subject Classification. Primary: 06D99. Secondary: 06C15, 03G25, 03G05.

DOI $10.46793 / \mathrm{KgJMat} 2106.977 \mathrm{G}$

Received: February 11, 2019.

Accepted: July 23, 2019. 
The concept of interval algebra was introduced in [9] for MV-algebras. Chajda and Kuhr extended this concept also for double basic algebras in [12]. In the case of a double basic algebra, they endowed the subinterval $[a, 1]$ with the structure of double basic algebra for all elements $a$ in a double basic algebra.

In this paper, we will obtain some properties of (pseudo commutative) double basic algebras. We find under what conditions pseudo commutative double basic algebras are double MV-algebras. We will study the relation between pseudo commutative double basic algebras and pseudo residuated l-gropoids. Finally, we will obtain some properties of boolean elements of a pseudo commutative double basic algebra and we will prove that a boolean center is subalgebra of a pseudo commutative double basic algebra. We will prove that, if $a, b$ are boolean elements of a pseudo commutative double basic algebra and $a \leq b$, then the subintervals of the form $[0, a]$ and $[a, b]$ can also be endowed with a double basic algebra structure.

\section{Preliminaries}

In this section, we recall some definitions and theorems which will be needed in this paper.

Definition 2.1 ([8,10]). A basic algebra is an algebra $(A, \oplus, \neg, 0,1)$ of type $(2,1,0,0)$ satisfying the following identities:

(BA1) $x \oplus 0=x$;

(BA2) $\neg \neg x=x$;

(BA3) $\neg(\neg x \oplus y) \oplus y=\neg(\neg y \oplus x) \oplus x$;

$(\mathrm{BA} 4) \neg(\neg(\neg(x \oplus y) \oplus y) \oplus z) \oplus(x \oplus z)=1$.

Orthomodular lattices and MV-algebras are examples of basic algebras. It is obvious that a basic algebra is an MV-algebra if and only if the operation $\oplus$ is associative and commutative. It was recently shown that a basic algebra is an MV-algebra if and only if it is associative (see [15]).

Definition $2.2([12])$. A double basic algebra is an algebra $A=\left(A, \oplus, \boxplus,^{-}, \sim, 0,1\right)$ of type $(2,2,1,1,0,0)$ satisfying the following identities:

(P1) $x \oplus 0=x, x \boxplus 0=x$;

(P2) $\left(x^{-}\right)^{\sim}=x,\left(x^{\sim}\right)^{-}=x$;

(P3) $\left(x^{\sim} \oplus y\right)^{-} \boxplus y=\left(y^{\sim} \oplus x\right)^{-} \boxplus x=\left(x^{-} \boxplus y\right)^{\sim} \oplus y=\left(y^{-} \boxplus x\right)^{\sim} \oplus x ;$

(P4) $\left(\left((x \boxplus y)^{\sim} \oplus y\right)^{-} \boxplus z\right)^{\sim} \oplus(x \boxplus z)=1,\left(\left((x \oplus y)^{-} \boxplus y\right)^{\sim} \oplus z\right)^{-} \boxplus(x \oplus z)=1$.

The connections between basic and double basic algebras are obvious. Suppose that $(A, \oplus, \neg, 0,1)$ is a basic algebra. Then $(A, \oplus, \oplus, \neg, \neg, 0,1)$ is a double basic algebra. Conversely, if we are given a double basic algebra in which $\oplus$ coincides with $\boxplus$, then the negations ${ }^{-}$and $\sim$ coincide too, hence the double basic algebra becomes a basic algebra (see [13]).

Proposition 2.1 ([13]). Let $\left(A, \oplus, \boxplus,^{-}, \sim, 0,1\right)$ be a double basic algebra. Then the reduct $\left(A, \boxplus,^{-}, 0,1\right)$ is a basic algebra if and only if $A$ satisfies the identity $x \oplus y=x \boxplus y$. 
We call a double basic algebra $\left(A, \oplus, \boxplus,^{-}, \sim, 0,1\right)$ pseudo-commutative if it satisfies the identity $x \oplus y=y \boxplus x$ for all $x, y \in A$.

Given a double basic algebra $\left(A, \oplus, \boxplus,^{-}, \sim, 0,1\right)$, define a binary relation $\leq$ on $A$ by $x \leq y$ if and only if $x^{\sim} \oplus y=1$ if and only if $x^{-} \boxplus y=1$. Then $(A, \leq)$ is a lattice and the following identities hold:

$$
x \vee y=\left(x^{\sim} \oplus y\right)^{-} \boxplus y \quad \text { and } \quad x \wedge y=\left(x^{-} \vee y^{-}\right)^{\sim}=\left(x^{\sim} \vee y^{\sim}\right)^{-} .
$$

If a double basic algebra is pseudo-commutative, then the induced lattice is distributive (see [12]).

Proposition $2.2([12,13])$. Let $\left(A, \oplus, \boxplus,^{-}, \sim, 0,1\right)$ be a double basic algebra. Then the following hold for all $x, y, z \in A$ :

(1) $0 \oplus x=x, 0 \boxplus x=x ;$

(2) $1 \oplus x=1=x \oplus 1,1 \boxplus x=1=x \boxplus 1$;

(3) $x^{\sim} \oplus x=1, x^{-} \boxplus x=1$;

(4) $x \leq y$ if and only if $y^{-} \leq x^{-}$if and only if $y^{\sim} \leq x^{\sim}$;

(5) $x \leq y$ implies $x \oplus z \leq y \oplus z$ and $x \boxplus z \leq y \boxplus z$;

(6) $y \leq x \oplus y$ and $y \leq x \boxplus y$;

(7) if $x \oplus y=z$ and $y \leq x^{-}$, then $x=\left(z^{-} \boxplus y\right)^{\sim}$;

(8) if $x \boxplus y=z$ and $y \leq x^{\sim}$, then $x=\left(z^{\sim} \oplus y\right)^{-}$;

(9) $\left(x \wedge y^{\sim}\right) \oplus y=x \oplus y,\left(x \wedge y^{-}\right) \boxplus y=x \boxplus y$;

(10) if $x^{-}, y$ are comparable, then $x \oplus y=x \oplus\left(y \wedge x^{-}\right)$;

(11) if $x^{\sim}, y$ are comparable, then $x \boxplus y=x \boxplus\left(y \wedge x^{\sim}\right)$;

(12) $(x \wedge y) \oplus z=(x \oplus z) \wedge(y \oplus z),(x \wedge y) \boxplus z=(x \boxplus z) \wedge(y \boxplus z)$;

(13) $1^{\sim}=0=1^{-}, 0^{\sim}=1=0^{-}$.

Let $\left(L, \wedge, \vee,\left(f_{a}\right)_{a \in L}, 0,1\right)$ be a bounded lattice equipped with a set $\left\{f_{a}, a \in L\right\}$ of partial mappings such that every $f_{a}$ is defined just on the section $[a, 1]$ and, moreover

(i) every $f_{a}$ is a bijection of $[a, 1]$ onto $[a, 1]$;

(ii) both $f_{a}$ and $f_{a}^{-1}$ are antitone, i.e., for $x, y \in[a, 1]$ with $x \leq y$, we have $f_{a}(y) \leq f_{a}(x)$ and $f_{a}^{-1}(y) \leq f_{a}^{-1}(x)$.

It was proved in [12] that to any bounded lattice with section antitone bijections can be assigned a double basic algebra. Also conversely, every double basic algebra induces a bounded lattice equipped with antitone bijections in every section.

Definition $2.3([12])$. A double MV-algebra is an algebra $\left(A, \oplus, \boxplus,^{-}, \sim, 0,1\right)$ of type $(2,2,1,1,0,0)$ satisfying the following identities for all $x, y, z \in A$ :

(D1) $x \oplus(y \oplus z)=(x \oplus y) \oplus z$ and $x \boxplus(y \boxplus z)=(x \boxplus y) \boxplus z ;$

(D2) $x \oplus y=y \boxplus x$;

(D3) $x \oplus 0=x$ and $x \boxplus 0=x$;

(D4) $x^{-\sim}=x$ and $x^{\sim-}=x$;

(D5) $x \oplus 1=1$ and $x \boxplus 1=1$;

(D6) $\left(x^{\sim} \oplus y\right)^{-} \boxplus y=\left(y^{\sim} \oplus x\right)^{-} \boxplus x=\left(x^{-} \boxplus y\right)^{\sim} \oplus y=\left(y^{-} \boxplus x\right)^{\sim} \oplus x$. 
Proposition $2.3([12])$. Let $\left(A, \oplus, \boxplus,^{-}, \sim, 0,1\right)$ be a double basic algebra. The following conditions are equivalent:

(a) $A$ is a double $M V$-algebra;

(b) $A$ is pseudo-commutative and associative both in $\oplus$ and $\boxplus$;

(c) A satisfies the Exchange identity $(x \oplus(y \boxplus z)=y \boxplus(x \oplus z))$.

Pseudo hoops were introduced by Bosbach in [2] and [3] under the name of complementary semigroups. It was proved that a bounded pseudo-hoop is a meet semilattice ordered residuated, integral and divisible monoid.

Definition $2.4([2])$. A pseudo-hoop is an algebra $(A, \odot, \rightarrow, \rightsquigarrow, 1)$ of type $(2,2,2,0)$ such that, for all $x, y, z \in A$ :

$($ psHOOP1) $x \odot 1=1 \odot x=x$;

(psHOOP2) $x \rightarrow x=x \rightsquigarrow x=1$;

$(\operatorname{psHOOP} 3)(x \odot y) \rightarrow z=x \rightarrow(y \rightarrow z)$;

$(\operatorname{psHOOP} 4)(x \odot y) \rightsquigarrow z=y \rightsquigarrow(x \rightsquigarrow z)$;

$($ psHOOP5) $(x \rightarrow y) \odot x=(y \rightarrow x) \odot y=x \odot(x \rightsquigarrow y)=y \odot(y \rightsquigarrow x)$.

Pseudo-BCK algebras were introduced by G. Georgescu and A. Iorgulescu ([19]) as non-commutative generalizations of BCK-algebras.

Definition 2.5 ([19]). A pseudo-BCK algebra (more precisely, reversed left-pseudo$\mathrm{BCK}$ algebra) is a structure $(A, \leq, \rightarrow, \rightsquigarrow, 1)$ where $\leq$ is a binary relation on $A, \rightarrow$ and $\rightsquigarrow$ are binary operations on $\mathrm{A}$ and 1 is an element of $\mathrm{A}$ satisfying, for all $x, y, z \in A$, the axioms:

$(\operatorname{psBCK} 1) x \rightarrow y \leq(y \rightarrow z) \rightsquigarrow(x \rightarrow z), x \rightsquigarrow y \leq(y \rightsquigarrow z) \rightarrow(x \rightsquigarrow z) ;$

$(\operatorname{psBCK} 2) x \leq(x \rightarrow y) \rightsquigarrow y, x \leq(x \rightsquigarrow y) \rightarrow y$;

$(\operatorname{psBCK} 3) x \leq x$

$(\mathrm{psBCK} 4) x \leq 1$

$(\operatorname{psBCK} 5)$ if $x \leq y$ and $y \leq x$, then $x=y$; (psBCK6) $x \leq y$ if and only if $x \rightarrow y=1$ if and only if $x \rightsquigarrow y=1$.

\section{Some Properties of Double Basic Algebras}

In any double basic algebra $\left(A, \oplus, \boxplus,^{-}, \sim, 0,1\right)$, we define four new derived binary operations as follows:

$$
\begin{aligned}
& x \odot y=\left(x^{\sim} \oplus y^{\sim}\right)^{-}, \quad x \boxminus y=\left(x^{-} \boxplus y^{-}\right)^{\sim}, \\
& x \rightsquigarrow y=x^{\sim} \oplus y, \quad x \rightarrow y=x^{-} \boxplus y,
\end{aligned}
$$

for all $x, y \in A$. It can be easily shown that

(C1) $x^{-}=x \rightarrow 0, x^{\sim}=x \rightsquigarrow 0$;

(C2) $x \oplus y=x^{-} \rightsquigarrow y, x \boxplus y=x^{\sim} \rightarrow y$;

(C3) $x \oplus y=\left(x^{-} \odot y^{-}\right)^{\sim}, x \boxplus y=\left(x^{\sim} \square y^{\sim}\right)^{-}$;

(C4) $x \vee y=(x \rightarrow y) \rightsquigarrow y=(y \rightarrow x) \rightsquigarrow x=(x \rightsquigarrow y) \rightarrow y=(y \rightsquigarrow x) \rightarrow x$;

$(\mathrm{C} 5) x \vee y=\left(x \odot y^{-}\right) \boxplus y=\left(y \odot x^{-}\right) \boxplus x=\left(x \boxminus y^{\sim}\right) \oplus y=\left(y \boxminus x^{\sim}\right) \oplus x$; 
(C6) $x \wedge y=\left(x \oplus y^{-}\right) \square y=\left(y \oplus x^{-}\right) \square x=\left(x \boxplus y^{\sim}\right) \odot y=\left(y \boxplus x^{\sim}\right) \odot x ;$

$(\mathrm{C} 7)(((x \rightsquigarrow y) \rightarrow y) \rightsquigarrow z) \rightarrow(x \rightsquigarrow z)=(((x \rightarrow y) \rightsquigarrow y) \rightarrow z) \rightsquigarrow(x \rightarrow z)=1$.

Proposition 3.1. Let $\left(A, \oplus, \boxplus,^{-}, \sim, 0,1\right)$ be a double basic algebra. Then for all $x, y, z \in A$, the following statements hold:

(1) $x \leq y$ if and only if $x \rightarrow y=1$ if and only if $x \rightsquigarrow y=1$;

(2) $x \rightarrow 1=x \rightarrow x=x \rightsquigarrow x=x \rightsquigarrow 1=1$;

(3) $1 \rightarrow x=1 \rightsquigarrow x=x$;

(4) $y \leq x \rightarrow y$ and $y \leq x \rightsquigarrow y$;

(5) $x \odot 1=1 \odot x=x, x \boxminus 1=1 \bullet x=x$;

(6) $x \odot y \leq y, x \boxminus y \leq y$;

(7) if $x \leq y$, then $x \odot z \leq y \odot z$ and $x \boxminus z \leq y \boxminus z$;

(8) $x \leq y$ implies that $y \rightarrow z \leq x \rightarrow z$ and $y \rightsquigarrow z \leq x \rightsquigarrow z$;

(9) $x \leq y \rightarrow z$ if and only if $y \leq x \rightsquigarrow z$;

(10) $((x \rightsquigarrow y) \rightarrow y) \rightsquigarrow y=x \rightsquigarrow y,((x \rightarrow y) \rightsquigarrow y) \rightarrow y=x \rightarrow y$;

(11) $(x \vee y) \rightarrow z=(x \rightarrow z) \wedge(y \rightarrow z),(x \vee y) \rightsquigarrow z=(x \rightsquigarrow z) \wedge(y \rightsquigarrow z)$.

Proof. The proof of parts (1)-(7) is straightforward by Proposition 2.2 and the definition of the operators.

(8) Suppose that $x \leq y$. Then $y^{\sim} \leq x^{\sim}$ by Proposition 2.2 part (4). Applying Proposition 2.2 part (5), we get $y^{\sim} \oplus z \leq x^{\sim} \oplus z$. By (P2) and (C2), we obtain $y \rightsquigarrow z \leq x \rightsquigarrow z$. Similarly, we can prove $y \rightarrow z \leq x \rightarrow z$.

(9) Suppose that $x \leq y \rightarrow z$. Using part (8), we have $(y \rightarrow z) \rightsquigarrow z \leq x \rightsquigarrow z$. By (C4), we have $y \leq y \vee z=(y \rightarrow z) \rightsquigarrow z$. Hence, $y \leq x \rightsquigarrow z$. Similarly, we can show that $y \leq x \rightsquigarrow z$ implies $x \leq y \rightarrow z$.

(10) Using (C4), we have $x \leq(x \rightsquigarrow y) \rightarrow y$ and $x \leq(x \rightarrow y) \rightsquigarrow y$. Applying part (8), we obtain $((x \rightsquigarrow y) \rightarrow y) \rightsquigarrow y \leq x \rightsquigarrow y$ and $((x \rightarrow y) \rightsquigarrow y) \rightarrow y \leq x \rightarrow y$. On the other hand, by (C4), we have $x \rightsquigarrow y \leq((x \rightsquigarrow y) \rightarrow y) \rightsquigarrow y$ and $x \rightarrow y \leq((x \rightarrow$ $y) \rightsquigarrow y) \rightarrow y$.

(11) We have that $x, y \leq x \vee y$. By part (8), we get $(x \vee y) \rightarrow z \leq x \rightarrow z$ and $(x \vee y) \rightarrow z \leq y \rightarrow z$. Thus $(x \vee y) \rightarrow z$ is a lower bound of $\{x \rightarrow z, y \rightarrow z\}$. Let $u$ be an arbitrary lower bound of $\{x \rightarrow z, y \rightarrow z\}$. Applying part (9), we get $x \leq u \rightsquigarrow z$ and $y \leq u \rightsquigarrow z$. Thus $x \vee y \leq u \rightsquigarrow z$. Using part (9), we have $u \leq(x \vee y) \rightarrow z$. Hence, $(x \vee y) \rightarrow z=(x \rightarrow z) \wedge(y \rightarrow z)$. Similarly, $(x \vee y) \rightsquigarrow z=(x \rightsquigarrow z) \wedge(y \rightsquigarrow z)$.

Proposition 3.2. Let $\left(A, \oplus, \boxplus,^{-}, \sim, 0,1\right)$ be a pseudo commutative double basic algebra. Then the following properties hold, for every $x, y, z \in A$ :

(1) $x \vee y \leq x \oplus y, x \vee y \leq x \boxplus y$;

(2) if $x \leq y$, then $z \rightarrow x \leq z \rightarrow y, z \rightsquigarrow x \leq z \rightsquigarrow y$;

(3) $x \rightarrow y=y^{-} \rightsquigarrow x^{-}, x \rightsquigarrow y=y^{\sim} \rightarrow x^{\sim}$;

(4) $x \rightarrow y^{\sim}=y \rightsquigarrow x^{-}$;

(5) if $x \leq y$, then $z \odot x \leq z \odot y, z \boxminus x \leq z \square y$;

(6) $(x \rightarrow y) \odot y^{-}=x^{-} \wedge y^{-},(x \rightsquigarrow y) \bullet y^{\sim}=x^{\sim} \wedge y^{\sim}$; 
(7) $(x \vee y) \odot z=(x \odot z) \vee(y \odot z),(x \vee y) \triangleright z=(x \boxminus z) \vee(y \boxminus z)$;

(8) $(x \rightarrow y) \odot x=x \wedge y=(x \rightsquigarrow y) \odot x$;

(9) $x \leq y \rightarrow z$ if and only if $x \square y \leq z$;

(10) $x \leq y \rightsquigarrow z$ if and only if $x \odot y \leq z$;

(11) $x \rightarrow(y \wedge z)=(x \rightarrow y) \wedge(x \rightarrow z), x \rightsquigarrow(y \wedge z)=(x \rightsquigarrow y) \wedge(x \rightsquigarrow z)$;

(12) $(y \vee z) \oplus x=(y \oplus x) \vee(z \oplus x),(y \vee z) \boxplus x=(y \boxplus x) \vee(z \boxplus x)$;

(13) $(x \wedge y) \odot z=(x \odot z) \wedge(y \odot z),(x \wedge y) \odot z=(x \boxminus z) \wedge(y \boxminus z)$.

Proof. The proofs of (1)-(4) are easy.

(5) Suppose $x \leq y$. By Proposition 2.2 part (4), we have $y^{-} \leq x^{-}$and $y^{\sim} \leq x^{\sim}$. Using Proposition 2.2 part (5), we obtain $y^{-} \oplus z^{-} \leq x^{-} \oplus z^{-}$and $y^{\sim} \boxplus z^{\sim} \leq x^{\sim} \boxplus z^{\sim}$. Since $A$ is pseudo commutative, then $z^{-} \boxplus y^{-} \leq z^{-} \boxplus x^{-}$and $z^{\sim} \oplus y^{\sim} \leq z^{\sim} \oplus x^{\sim}$. By Proposition 2.2 part (4), we have $\left(z^{-} \boxplus x^{-}\right)^{\sim} \leq\left(z^{-} \boxplus y^{-}\right)^{\sim}$ and $\left(z^{\sim} \oplus x^{\sim}\right)^{-} \leq\left(z^{\sim} \oplus y^{\sim}\right)^{-}$, that is $z \odot x \leq z \odot y, z \boxminus x \leq z \square y$.

(6) We have $(x \rightarrow y) \odot y^{-}=\left(\left(x^{-} \boxplus y\right)^{\sim} \oplus y\right)^{-}=(x \vee y)^{-}=x^{-} \wedge y^{-}$. Similarly, we can prove $(x \rightarrow y) \square y^{\sim}=x^{\sim} \wedge y^{\sim}$.

(7) It follows from Proposition 2.2 part (12), (P2) and definition $\odot$.

(8) Since $A$ is pseudo commutative and by (C6), we have

$$
\begin{aligned}
& (x \rightarrow y) \triangleright x=\left(x^{-} \boxplus y\right) \bullet x=\left(y \oplus x^{-}\right) \bullet x=x \wedge y \\
& (x \rightsquigarrow y) \odot x=\left(x^{\sim} \oplus y\right) \odot x=\left(y \boxplus x^{\sim}\right) \odot x=x \wedge y .
\end{aligned}
$$

(9) Let $x \leq y \rightarrow z$. By Proposition 3.1 part (7), we get $x \boxminus y \leq(y \rightarrow z) \square y=$ $y \wedge z \leq z$. Conversely, suppose $x \boxminus y \leq z$. Then

$$
x \leq\left(x \vee y^{-}\right)=(x \boxminus y) \oplus y^{-} \leq z \oplus y^{-}=y^{-} \boxplus z=y \rightarrow z .
$$

(10) The proof is similar to part (9).

(11) Since $y \wedge z \leq y, z$, then $x \rightarrow(y \wedge z) \leq x \rightarrow y$ and $x \rightarrow(y \wedge z) \leq x \rightarrow z$ by part (2). Hence, $x \rightarrow(y \wedge z)$ is a lower bound of $\{x \rightarrow y, x \rightarrow z\}$. Let $u$ be an arbitrary lower bound of $\{x \rightarrow y, x \rightarrow z\}$. Then $u \leq x \rightarrow y$ and $u \leq x \rightarrow z$. By part (9), we have $u \boxminus x \leq y$ and $u \boxminus x \leq z$. So, $u \boxminus x \leq y \wedge z$. Again, applying part (9), we obtain $u \leq x \rightarrow(y \wedge z)$. Hence, $x \rightarrow(y \wedge z)=(x \rightarrow y) \wedge(x \rightarrow z)$. Similarly, $x \rightsquigarrow(y \wedge z)=(x \rightsquigarrow y) \wedge(x \rightsquigarrow z)$.

(12) Since $y, z \leq y \vee z$, then $y \oplus x \leq(y \vee z) \oplus x$ and $z \oplus x \leq(y \vee z) \oplus x$ by Proposition 2.4 part (5). Hence, $(y \oplus x) \vee(z \oplus x) \leq(y \vee z) \oplus x$. Conversely, let $u:=(y \oplus x) \vee(z \oplus x)$. Then $y \oplus x \leq u$ and $z \oplus x \leq u$. By Proposition 3.1 part (7) and (C6), we have $z \wedge x^{\sim}=(z \oplus x) \boxminus x^{\sim} \leq u \boxminus x^{\sim}$ and $y \wedge x^{\sim}=(y \oplus x) \boxminus x^{\sim} \leq u \boxminus x^{\sim}$. Since $A$ is a distributive lattice, then $(z \vee y) \wedge x^{\sim}=\left(y \wedge x^{\sim}\right) \vee\left(z \wedge x^{\sim}\right) \leq u \boxminus x^{\sim}$. By Proposition 2.2 part (5) and (C5), we get $\left((z \vee y) \wedge x^{\sim}\right) \oplus x \leq\left(u \boxminus x^{\sim}\right) \oplus x=u \vee x=u$. Using Proposition 2.2 part (12) and part (3), we obtain $((z \vee y) \oplus x) \wedge\left(x^{\sim} \oplus x\right)=(z \vee y) \oplus x \leq u$. Hence, $(z \vee y) \oplus x \leq(y \oplus x) \vee(z \oplus x)$.

(13) It follows from part (12) and definitions $\odot$ and $\odot$.

Corollary 3.1. Let $\left(A, \oplus, \boxplus,^{-}, \sim, 0,1\right)$ be a pseudo commutative double basic algebra. Then $y \odot x=x \boxminus y$ for all $x, y \in A$. 
Proof. Since $x \boxminus y \leq x \boxminus y$, then $x \leq y \rightarrow(x \boxminus y)$ by Proposition 3.2 part (9). We obtain $y \leq x \rightsquigarrow(x \boxminus y)$ by Proposition 3.1 part (9). Using Proposition 3.2 part (10), we get $y \odot x \leq x \boxminus y$. Similarly, we can prove $x \bullet y \leq y \odot x$. Hence $x \boxminus y=y \odot x$.

Proposition 3.3. Let $\left(A, \oplus, \boxplus,^{-}, \sim, 0,1\right)$ be a pseudo commutative double basic algebra.

(1) If $(x \odot y) \rightsquigarrow z=x \rightsquigarrow(y \rightsquigarrow z)$ for any $x, y, z \in A$, then $\oplus$ is associative.

(2) If $(x \boxminus y) \rightarrow z=x \rightarrow(y \rightarrow z)$ for any $x, y, z \in A$, then $\boxplus$ is associative.

Proof. (1) We have $\left(x^{\sim} \oplus y^{\sim}\right) \oplus z=(x \odot y) \rightsquigarrow z=x \rightsquigarrow(y \rightsquigarrow z)=x^{\sim} \oplus\left(y^{\sim} \oplus z\right)$. Hence $\oplus$ is associative. The proof of part (2) is similar.

Corollary 3.2. Let $\left(A, \oplus, \boxplus,^{-}, \sim, 0,1\right)$ be a pseudo commutative double basic algebra such that $(A, \wedge, \vee, \odot, \rightsquigarrow, \rightarrow, 1)$ and $(A, \wedge, \vee, \odot, \rightarrow, \rightsquigarrow, 1)$ be pseudo hoops. Then $A$ is a double $M V$-algebra.

Proof. Let $(A, \wedge, \vee, \odot, \rightsquigarrow, \rightarrow, 1)$ and $(A, \wedge, \vee, \odot, \rightarrow, \rightsquigarrow, 1)$ be pseudo hoops. Then $\oplus$ and $\boxplus$ are associative by Proposition 3.3. Since $\oplus$ and $\boxplus$ are associative and $A$ is a pseudo commutative, then $A$ is a double MV-algebra.

Proposition 3.4. Let $\left(A, \oplus, \boxplus,^{-}, \sim, 0,1\right)$ be a pseudo commutative double basic algebra such that satisfies the following identities for all $x, y, z \in A$

$$
x \rightarrow y \leq(y \rightarrow z) \rightsquigarrow(x \rightarrow z), \quad x \rightsquigarrow y \leq(y \rightsquigarrow z) \rightarrow(x \rightsquigarrow z) .
$$

Then $\oplus$ and $\boxplus$ are associative.

Proof. Suppose that $A$ satisfies $x \rightarrow y \leq(y \rightarrow z) \rightsquigarrow(x \rightarrow z)$ and $x \rightsquigarrow y \leq(y \rightsquigarrow z) \rightarrow$ $(x \rightsquigarrow z)$. Then $(A, \leq, \rightarrow, \rightsquigarrow, 1)$ is a pseudo-BCK algebra. By Proposition 1.2 part (3) in [17], we have $x \rightsquigarrow(y \rightarrow z)=y \rightarrow(x \rightsquigarrow z)$ and $x \rightarrow(y \rightsquigarrow z)=y \rightsquigarrow(x \rightarrow z)$. Thus, $x^{\sim} \oplus\left(y^{-} \boxplus z\right)=y^{-} \boxplus\left(x^{\sim} \oplus z\right)$. So $A$ satisfies Exchange identity. Hence, $\oplus$ and $\boxplus$ are associative by Proposition 2.3 .

Corollary 3.3. Let $\left(A, \oplus, \boxplus,^{-}, \sim, 0,1\right)$ be a pseudo commutative double basic algebra such that $(A, \leq, \rightarrow, \rightsquigarrow, 1)$ is a pseudo BCK-algebra. Then $A$ is a double MV-algebra.

Proof. Similarly to the proof of Proposition 3.4, we can prove the associativity of $\oplus$ and $\boxplus$. Since $A$ is a pseudo commutative, then $A$ is a double MV-algebra by Proposition 2.3.

The relation between (commutative) basic algebras and residuated groupoids have been studied in [7]. In the following, we will study the relation between pseudo commutative double basic algebras and pseudo residuated l-groupoids.

Definition 3.1. A pseudo residuated $l$-groupoid is an algebra $(A, \wedge, \vee, *, \rightarrow, \rightsquigarrow, 0,1)$ of type $(2,2,2,2,2,0,0)$ such that

(R1) $(A, \wedge, \vee, 0,1)$ is a bounded lattice;

(R2) $(A, *, 1)$ is a groupoid with 1 , i.e. it satisfies $x * 1=1 * x=x$; 
(R3) $x * y \leq z$ if and only if $x \leq y \rightarrow z$ if and only if $y \leq x \rightsquigarrow z$ for any $x, y, z \in A$ (pseudo residuation).

Proposition 3.5. Let $(A, \wedge, \vee, *, \rightarrow, \rightsquigarrow, 0,1)$ be a pseudo residuated l-groupoid. Then

(1) if $x \leq y$, then $x * z \leq y * z$ and $z * x \leq z * y$;

(2) $(x \rightarrow y) * x \leq x \wedge y, x *(x \rightsquigarrow y) \leq x \wedge y$;

(3) $x \leq y$ implies $y \rightarrow z \leq x \rightarrow z$ and $y \rightsquigarrow z \leq x \rightsquigarrow z$;

(4) $1 \rightarrow x=x, 1 \rightsquigarrow x=x$.

Proof. (1) Suppose that $x \leq y$. Since $y * z \leq y * z$ and $z * y \leq z * y$, then $y \leq z \rightarrow y * z$ and $y \leq z \rightsquigarrow z * y$. Thus $x \leq z \rightarrow y * z$ and $x \leq z \rightsquigarrow z * y$. Therefore $x * z \leq y * z$ and $z * x \leq z * y$.

The proofs of (2)-(4) are easy.

Proposition 3.6. Let $\left(A, \oplus, \boxplus,^{-}, \sim, 0,1\right)$ be a pseudo commutative double basic algebra. Then $(A, \wedge, \vee, \odot, \rightarrow, \rightsquigarrow, 0,1)$ and $(A, \wedge, \vee, \odot, \rightsquigarrow, \rightarrow, 0,1)$ are pseudo residuated l-groupoids.

Proof. Since $A$ is a pseudo commutative double basic algebra, then $(A, \wedge, \vee, 0,1)$ is a bounded distributive lattice. By Proposition 3.1 part $(5),(A, \odot, 1)$ and $(A, \odot, 1)$ are groupoids with 1. Applying Proposition 3.2 part (9) and Proposition 3.1 part (9), we get $x \square y \leq z$ if and only if $x \leq y \rightarrow z$ if and only if $y \leq x \rightsquigarrow z$.

Hence, $(A, \wedge, \vee, \square, \rightarrow, \rightsquigarrow, 0,1)$ is a pseudo residuated $l$-groupoid.

Again, By Proposition 3.2 part (10) and Proposition 3.1 part (9), we have $x \odot y \leq z$ if and only if $x \leq y \rightsquigarrow z$ if and only if $y \leq x \rightarrow z$.

Hence, $(A, \wedge, \vee, \odot, \rightsquigarrow, \rightarrow, 1)$ is a pseudo residuated l-groupoid.

For the case of basic algebra, the next theorems are proved in [7]. Here, we formulate and prove them for the case of pseudo commutative double basic algebras as well.

Lemma 3.1. Let $(A, \wedge, \vee, *, \rightarrow, \rightsquigarrow, 0,1)$ be a pseudo residuated l-groupoid satisfying the pseudo double negation laws and

(i) $x *\left(y^{\sim} \rightarrow x^{\sim}\right)=x \wedge y$;

(ii) $\left(y^{-} \rightsquigarrow x^{-}\right) * x=x \wedge y$;

then the following hold:

(1) $x \rightarrow y=y^{-} \rightsquigarrow x^{-}, x \rightsquigarrow y=y^{\sim} \rightarrow x^{\sim}$;

(2) $x *(y * z)=0$ implies $(x * y) * z=0$;

(3) $x \rightarrow y=\left(x * y^{\sim}\right)^{-}, x \rightsquigarrow y=\left(y^{-} * x\right)^{\sim}$;

where $x^{-}=x \rightarrow 0$ and $x^{\sim}=x \rightsquigarrow 0$.

Proof. (1) By assumption, we have $x *\left(y^{\sim} \rightarrow x^{\sim}\right)=x \wedge y \leq y$ and $\left(y^{-} \rightsquigarrow x^{-}\right) * x=$ $x \wedge y \leq y$. Thus, $y^{\sim} \rightarrow x^{\sim} \leq x \rightsquigarrow y$ and $y^{-} \rightsquigarrow x^{-} \leq x \rightarrow y$.

Using the pseudo double negation laws and substituting $y^{-}$for $x$ and $x^{-}$for $y$ in $y^{\sim} \rightarrow x^{\sim} \leq x \rightsquigarrow y$, this yields $x \rightarrow y \leq y^{-} \rightsquigarrow x^{-}$. 
In $y^{-} \rightsquigarrow x^{-} \leq x \rightarrow y$, applying the pseudo double negation laws and substituting $y^{\sim}$ for $x$ and $x^{\sim}$ for $y$, this yields $x \rightsquigarrow y \leq y^{\sim} \rightarrow x^{\sim}$. Hence, $x \rightarrow y=y^{-} \rightsquigarrow x^{-}$and $x \rightsquigarrow y=y^{\sim} \rightarrow x^{\sim}$.

(2) Suppose $x *(y * z)=0$. Then $y * z \leq x \rightsquigarrow 0=x^{\sim}$. So, $y \leq z \rightarrow x^{\sim}$. By part (1) and pseudo double negation laws, we get $y \leq x \rightsquigarrow z^{-}$. Thus, $x * y \leq z \rightarrow 0$. Hence, $(x * y) * z=0$.

(3) Since $x * y^{\sim} \leq x * y^{\sim}$, then $x \leq y^{\sim} \rightarrow\left(x * y^{\sim}\right)$. Applying part (1) and pseudo double negation laws, we have $x \leq\left(x * y^{\sim}\right)^{-} \rightsquigarrow y$. So, $\left(x * y^{\sim}\right)^{-} * x \leq y$. Hence, $\left(x * y^{\sim}\right)^{-} \leq x \rightarrow y$.

On the other hand, using part (1) and then assumption (ii), we obtain

$$
((x \rightarrow y) * x) * y^{\sim}=\left(\left(y^{-} \rightsquigarrow x^{-}\right) * x\right) * y^{\sim}=(x \wedge y) * y^{\sim} \leq y * y^{\sim}=0 .
$$

Hence, $((x \rightarrow y) * x) * y^{\sim}=0$. By part $(2)$, we get $(x \rightarrow y) *\left(x * y^{\sim}\right)=0$. Thus, $x \rightarrow y \leq\left(x * y^{\sim}\right)^{-}$. Therefore, $x \rightarrow y=\left(x * y^{\sim}\right)^{-}$. Similarly, we can prove $x \rightsquigarrow y=\left(y^{-} * x\right)^{\sim}$.

Theorem 3.1. Let $(A, \wedge, \vee, *, \rightarrow, \rightsquigarrow, 0,1)$ be a pseudo residuated l-groupoid satisfying the conditions of Lemma 3.1. Then $(A, \oplus, \boxplus,-, \sim, 0,1)$ is a pseudo commutative double basic algebra where $x^{-}=x \rightarrow 0, x^{\sim}=x \rightsquigarrow 0, x \oplus y=\left(y^{-} * x^{-}\right)^{\sim}$ and $x \boxplus y=\left(x^{\sim} * y^{\sim}\right)^{-}$.

Proof. We will check the axioms (P1)-(P4).

(P1) We have $x \oplus 0=\left(0^{-} * x^{-}\right)^{\sim}=x^{-\sim}=x$ and $x \boxplus 0=\left(x^{\sim} * 0^{\sim}\right)^{-}=x^{\sim-}=x$.

(P2) It holds by assumption (pseudo double negation laws).

(P3) By Lemma 3.1 part (3), definitions of $\oplus$ and $\boxplus$, pseudo double negation laws and then assumption (i), we get

$$
\begin{aligned}
\left(x^{\sim} \oplus y\right)^{-} \boxplus y & =\left(y^{-} * x\right) \boxplus y=(x \rightsquigarrow y)^{-} \boxplus y=\left((x \rightsquigarrow y) * y^{\sim}\right)^{-} \\
& =\left(\left(x^{\sim-} \rightsquigarrow y^{\sim-}\right) * y^{\sim}\right)^{-}=\left(x^{\sim} \wedge y^{\sim}\right)^{-}=x \vee y .
\end{aligned}
$$

Similarly, we can prove $\left(y^{\sim} \oplus x\right)^{-} \boxplus x=\left(x^{-} \boxplus y\right)^{\sim} \oplus y=\left(y^{-} \boxplus x\right)^{\sim} \oplus x=x \vee y$.

(P4) It is easy to prove $\left(x \wedge y^{-}\right) \boxplus z \leq x \boxplus z$. Hence,

$$
\begin{aligned}
1 & =\left(\left(x \wedge y^{-}\right) \boxplus z\right) \rightsquigarrow(x \boxplus z) \\
& =\left(\left(y^{-} *\left(x^{\sim} \rightarrow y^{-\sim}\right)\right) \boxplus z\right) \rightsquigarrow(x \boxplus z) \\
& =\left(\left(y^{-} *\left(x^{\sim} \rightarrow y\right)\right) \boxplus z\right) \rightsquigarrow(x \boxplus z) \\
& =\left(\left(y^{-} *(x \boxplus y)\right) \boxplus z\right) \rightsquigarrow(x \boxplus z) \\
& =\left(\left((x \boxplus y)^{\sim} \oplus y\right)^{-} \boxplus z\right) \rightsquigarrow(x \boxplus z) \\
& =\left(\left((x \boxplus y)^{\sim} \oplus y\right)^{-} \boxplus z\right)^{\sim} \oplus(x \boxplus z) .
\end{aligned}
$$

Similarly, we can prove $\left(\left((x \oplus y)^{-} \boxplus y\right)^{\sim} \oplus z\right)^{-} \boxplus(x \oplus z)=1$. Hence, $\left(A, \oplus, \boxplus,^{-}, \sim, 0,1\right)$ is a double basic algebra. By Lemma 3.11 part (1) and (3), we have

$$
x \oplus y=\left(y^{-} * x^{-}\right)^{\sim}=x^{-} \rightsquigarrow y=y^{\sim} \rightarrow x=\left(y^{\sim} * x^{\sim}\right)^{-}=y \boxplus x .
$$

Hence, $\left(A, \oplus, \boxplus,^{-}, \sim, 0,1\right)$ is a pseudo commutative. 
Lemma 3.2. Let $(A, \wedge, \vee, *, \rightarrow, \rightsquigarrow, 0,1)$ be a pseudo residuated l-groupoid satisfying the following identities:

$$
x \vee y=\left(y^{-} \rightsquigarrow x^{-}\right) \rightsquigarrow y=\left(y^{\sim} \rightarrow x^{\sim}\right) \rightarrow y ;
$$

then the following hold:

(1) $x^{-\sim}=x, x^{\sim-}=x$;

(2) $x \rightarrow y=y^{-} \rightsquigarrow x^{-}, x \rightsquigarrow y=y^{\sim} \rightarrow x^{\sim}$;

(3) $x *(y * z)=0$ implies $(x * y) * z=0$;

(4) $x \rightarrow y=\left(x * y^{\sim}\right)^{-}, x \rightsquigarrow y=\left(y^{-} * x\right)^{\sim}$;

(5) $(x \rightarrow y) * x=x \wedge y, x *(x \rightsquigarrow y)=x \wedge y$.

Proof. (1) We have $1 \rightsquigarrow x^{-}=x^{-} \leq x \rightarrow 0$ by Proposition 3.5 part (4). Applying Proposition 3.5 part (3) and assumption, we get $(x \rightarrow 0) \rightsquigarrow 0 \leq\left(1 \rightsquigarrow x^{-}\right) \rightsquigarrow 0=$ $x \vee 0=x$. Hence, $x^{-\sim} \leq x$.

On the other hand, $(x \rightarrow 0) * x \leq 0$ by Proposition 3.5 part (2). Hence, $x \leq(x \rightarrow$ 0) $\rightsquigarrow 0=x^{-\sim}$. Therefore, $x^{-\sim}=x$. Similarly, we can prove $x^{\sim-}=x$.

(2) Since $x \leq x \vee y=\left(y^{-} \rightsquigarrow x^{-}\right) \rightsquigarrow y$, then $\left(y^{-} \rightsquigarrow x^{-}\right) * x \leq y$. Thus, $y^{-} \rightsquigarrow x^{-} \leq$ $x \rightarrow y$. Also, using the pseudo double negation laws and substituting $y^{\sim}$ for $x$ and $x^{\sim}$ for $y$ in $y^{-} \rightsquigarrow x^{-} \leq x \rightarrow y$, we obtain $x \rightsquigarrow y \leq y^{\sim} \rightarrow x^{\sim}$.

Similarly, we can show $y^{\sim} \rightarrow x^{\sim} \leq x \rightsquigarrow y$ and $x \rightarrow y \leq y^{-} \rightsquigarrow x^{-}$. Hence, $x \rightarrow y=y^{-} \rightsquigarrow x^{-}, x \rightsquigarrow y=y^{-} \rightarrow x^{-}$.

The proof of part (3) and part (4) is similar to the proof of part (2) and part (3) in Lemma 3.1, respectively.

(5) Using part (1), part (4) and then assumption, we get

$$
(x \rightarrow y) * x=\left((x \rightarrow y) * x^{-\sim}\right)^{-\sim}=\left((x \rightarrow y) \rightarrow x^{-}\right)^{\sim}=\left(x^{-} \vee y^{-}\right)^{\sim}=x \wedge y .
$$

Similarly, we can prove that $x *(x \rightsquigarrow y)=x \wedge y$.

Proposition 3.7. Let $(A, \wedge, \vee, *, \rightarrow, \rightsquigarrow, 0,1)$ be a pseudo residuated l-groupoid. Then A satisfies the conditions of Lemma 3.2 if and only if it satisfies the conditions of Lemma 3.1.

Proof. Let $(A, \wedge, \vee, *, \rightarrow, \rightsquigarrow, 0,1)$ be a pseudo residuated $l$-groupoid satisfying conditions of Lemma 3.1. Using Lemma 3.1 part (1) and part (3), we obtain

$$
\begin{aligned}
& x \vee y=\left(y^{-} \wedge x^{-}\right)^{\sim}=\left(y^{-} *(x \rightarrow y)\right)^{\sim}=(x \rightarrow y) \rightsquigarrow y=\left(y^{-} \rightsquigarrow x^{-}\right) \rightsquigarrow y ; \\
& x \vee y=\left(y^{\sim} \wedge x^{\sim}\right)^{-}=\left((x \rightarrow y) * y^{\sim}\right)^{-}=(x \rightsquigarrow y) \rightarrow y=\left(y^{\sim} \rightarrow x^{\sim}\right) \rightarrow y .
\end{aligned}
$$

Conversely, suppose that $A$ satisfies the conditions of Lemma 3.2. Applying Lemma 3.2 part (2) and then part (5), we obtain

$$
x *\left(y^{\sim} \rightarrow x^{\sim}\right)=x *(x \rightsquigarrow y)=x \wedge y, \quad\left(y^{-} \rightsquigarrow x^{-}\right) * x=(x \rightarrow y) * x=x \wedge y .
$$

Also, by part (1) of Lemma 3.2, $A$ satisfies the pseudo double negation laws.

Corollary 3.4. Let $(A, \wedge, \vee, *, \rightarrow, \rightsquigarrow, 0,1)$ be a pseudo residuated l-groupoid satisfying the following identities: 


$$
x \vee y=\left(y^{-} \rightsquigarrow x^{-}\right) \rightsquigarrow y=\left(y^{\sim} \rightarrow x^{\sim}\right) \rightarrow y .
$$

Then $\left(A, \oplus, \boxplus,^{-}, \sim, 0,1\right)$ is a pseudo commutative double basic algebra where $x^{-}=$ $x \rightarrow 0, x^{\sim}=x \rightsquigarrow 0, x \oplus y=\left(y^{-} * x^{-}\right)^{\sim}$ and $x \boxplus y=\left(x^{\sim} * y^{\sim}\right)^{-}$.

Proof. The proof follows from Proposition 3.7 and Theorem 3.1.

By Proposition 3.6, if $\left(A, \oplus, \boxplus,^{-}, \sim, 0,1\right)$ is a pseudo commutative double basic algebra, then $F\left(\left(A, \oplus, \boxplus,^{-}, \sim, 0,1\right)\right):=(A, \wedge, \vee, \square, \rightarrow, \rightsquigarrow, 0,1)$ is a pseudo residuated $l$-groupoid where $x \boxminus y=\left(x^{-} \boxplus y^{-}\right)^{\sim}, x \rightarrow y=\left(x \boxminus y^{\sim}\right)^{-}, x \rightsquigarrow y=\left(y^{-} \boxminus x\right)^{\sim}$.

Moreover, if $(A, \wedge, \vee, *, \rightarrow, \rightsquigarrow, 0,1)$ is a pseudo residuated $l$-groupoid that satisfies the conditions of Lemma 3.1 (or Lemma 3.2 ), then $G((A, \wedge, \vee, *, \rightarrow, \rightsquigarrow, 0,1)):=$ $\left(A, \oplus, \boxplus,^{-}, \sim, 0,1\right)$ is a pseudo commutative double basic algebra, where $x^{-}=x \rightarrow 0$, $x^{\sim}=x \rightsquigarrow 0, x \oplus y=\left(y^{-} * x^{-}\right)^{\sim}$ and $x \boxplus y=\left(x^{\sim} * y^{\sim}\right)^{-}$.

The category whose objects are double basic algebras and whose morphisms are homomorphisms of double basic algebras is called the category of double basic algebras. Let $\mathcal{A}$ be its subcategory whose object are pseudo commutative double basic algebras. The category of pseudo residuated $l$-groupoids can be defined similarly. Let $\mathcal{B}$ be its subcategory whose objects are pseudo residuated l-groupoids satisfying conditions of Lemma 3.1. It is clear that $F: \mathcal{A} \rightarrow \mathcal{B}$ and $G: \mathcal{B} \rightarrow \mathcal{A}$ are functors. In the next theorem, we study a relation between these functors.

Theorem 3.2. (1) Let $\left(A, \oplus, \boxplus,^{-}, \sim, 0,1\right)$ be a pseudo commutative double basic algebra. Then $G\left(F\left(\left(A, \oplus, \boxplus,^{-}, \sim, 0,1\right)\right)\right)=\left(A, \oplus, \boxplus,^{-}, \sim, 0,1\right)$.

(2) Let $(A, \wedge, \vee, *, \rightarrow, \rightsquigarrow, 0,1)$ be a pseudo residuated l-groupoid that satisfies the conditions of Lemma 3.1. Then

$$
F(G((A, \wedge, \vee, *, \rightarrow, \rightsquigarrow, 0,1)))=(A, \wedge, \vee, *, \rightarrow, \rightsquigarrow, 0,1) .
$$

(3) The category $\mathcal{A}$ and the category $\mathcal{B}$ are categorically isomorphic.

Proof. (1) By Proposition 3.6, Proposition 3.2 part (3) and part (8),

$$
F\left(\left(A, \oplus, \boxplus,^{-}, \sim, 0,1\right)\right):=(A, \wedge, \vee, \square, \rightarrow, \rightsquigarrow, 0,1)
$$

is a pseudo residuated $l$-groupoid satisfying conditions of Lemma 3.1. Hence, $G\left(F\left(\left(A, \oplus, \boxplus,^{-}, \sim, 0,1\right)\right)\right)$ is a pseudo commutative double basic algebra by Theorem 3.1. Now, suppose that $\oplus^{\prime}, \boxplus^{\prime},{{ }^{\prime}}^{\prime}$ and $\sim^{\prime}$ are the operations derived by $\square, \rightarrow$ and $\rightsquigarrow$ on the pseudo residuated $l$-groupoid $F\left(\left(A, \oplus, \boxplus,,^{-}, \sim, 0,1\right)\right)$, respectively. We will prove that $\oplus^{\prime}=\oplus, \boxplus^{\prime}=\boxplus,{ }^{-^{\prime}}={ }^{-}$and $\sim^{\prime}=\sim$. Let $x, y \in A$ be arbitrary. We have $x^{-^{\prime}}=x \rightarrow 0=x^{-} \boxplus 0=x^{-}$and $x^{\sim^{\prime}}=x \rightsquigarrow 0=x^{\sim} \oplus 0=x^{\sim}$. Using Lemma 3.1 part

(3) and part (1), we get

$$
\begin{aligned}
& x \boxplus^{\prime} y=\left(x^{\sim^{\prime}} \square y^{\sim^{\prime}}\right)^{-^{\prime}}=\left(x^{\sim} \square y^{\sim}\right)^{-}=x \boxplus y, \\
& x \oplus^{\prime} y=\left(x^{-^{\prime}} \square y^{-^{\prime}}\right)^{\sim^{\prime}}=\left(y^{-} \boxminus x^{-}\right)^{\sim}=x^{-} \rightsquigarrow y=y^{\sim} \rightarrow x=\left(y^{\sim} \odot x^{\sim}\right)^{-}=y \boxplus x \\
& =x \oplus y \text {. }
\end{aligned}
$$


(2) By Theorem 3.1, $G((A, \wedge, \vee, *, \rightarrow, \rightsquigarrow, 0,1)):=\left(A, \oplus, \boxplus,^{-}, \sim, 0,1\right)$ is a pseudo commutative double basic algebra. Hence, $F(G((A, \wedge, \vee, *, \rightarrow, \rightsquigarrow, 0,1)))$ is a pseudo residuated $l$-groupoid satisfying conditions of Lemma 3.1. Let $F(G((A, \wedge, \vee, *, \rightarrow, \rightsquigarrow$ $, 0,1)))=\left(A, \wedge^{\prime}, \vee^{\prime}, *^{\prime}, \rightarrow^{\prime}, \rightsquigarrow^{\prime}, 0,1\right)$. We have

$$
\begin{aligned}
x *^{\prime} y & =x \boxminus y=\left(x^{-} \boxplus y^{-}\right)^{\sim}=\left(x^{-\sim} * y^{-\sim}\right)^{-\sim}=x * y, \\
x \rightarrow{ }^{\prime} y & =x^{-} \boxplus y=\left(x * y^{\sim}\right)^{-}=x \rightarrow y, \\
x \rightsquigarrow^{\prime} y & =x^{\sim} \oplus y=\left(y^{-} * x\right)^{-}=x \rightsquigarrow y, \\
x \wedge^{\prime} y & =x *^{\prime}\left(y^{\sim^{\prime}} \rightarrow x^{\sim^{\prime}}\right)=x *\left(y^{\sim} \rightarrow x^{\sim}\right)=x \wedge y, \\
x \vee^{\prime} y & =\left(x^{-^{\prime}} \wedge^{\prime} y^{-^{\prime}}\right)^{\sim^{\prime}}=\left(x^{-} \wedge y^{-}\right)^{\sim} .
\end{aligned}
$$

(3) The proof follows from (i) and (ii).

By Proposition 3.6, if $\left(A, \oplus, \boxplus,^{-}, \sim, 0,1\right)$ is a pseudo commutative double basic algebra, then $H\left(\left(A, \oplus, \boxplus,^{-}, \sim, 0,1\right)\right):=(A, \wedge, \vee, \odot, \rightsquigarrow, \rightarrow, 0,1)$ is a pseudo residuated l-groupoid, (that is $x \odot y \leq z$ if and only if $x \leq y \rightsquigarrow z$ if and only if $y \leq x \rightarrow z$ for any $x, y, z \in A)$ where $x \odot y=\left(x^{\sim} \oplus y^{\sim}\right)^{-}, x \rightsquigarrow y=x^{\sim} \oplus y, x \rightarrow y=y \oplus x^{-}$. Then clearly, $H: \mathcal{A} \rightarrow \mathcal{B}$ is a functor.

Proposition 3.8. Let $(A, \wedge, \vee, *, \rightsquigarrow, \rightarrow, 0,1)$ be a pseudo residuated l-groupoid satisfying pseudo double negation laws and

(i)' $\left(y^{\sim} \rightarrow x^{\sim}\right) * x=x \wedge y$;

(ii)' $x *\left(y^{-} \rightsquigarrow x^{-}\right)=x \wedge y$.

Then $\left(A, \oplus, \boxplus,^{-}, \sim, 0,1\right)$ is a pseudo commutative double basic algebra, where $x^{-}=$ $x \rightarrow 0, x^{\sim}=x \rightsquigarrow 0, x \oplus y=\left(x^{-} * y^{-}\right)^{\sim}$ and $x \boxplus y=\left(y^{\sim} * x^{\sim}\right)^{-}$.

Proof. Similar to Lemma 3.1, we can prove

(1) $x \rightarrow y=y^{-} \rightsquigarrow x^{-}, x \rightsquigarrow y=y^{\sim} \rightarrow x^{\sim}$;

(2) $x *(y * z)=0$ implies $(x * y) * z=0$;

(3) $x \rightarrow y=\left(y^{\sim} * x\right)^{-}, x \rightsquigarrow y=\left(x * y^{-}\right)^{\sim}$;

and use them to prove that $\left(A, \oplus, \boxplus,^{-}, \sim, 0,1\right)$ is a pseudo commutative double basic algebra such that $x^{-}=x \rightarrow 0, x^{\sim}=x \rightsquigarrow 0, x \oplus y=\left(x^{-} * y^{-}\right)^{\sim}$ and $x \boxplus y=$ $\left(y^{\sim} * x^{\sim}\right)^{-}$.

Theorem 3.3. (1) Let $\left(A, \oplus, \boxplus,^{-}, \sim, 0,1\right)$ be a pseudo commutative double basic algebra. Then $G\left(H\left(\left(A, \oplus, \boxplus,^{-}, \sim, 0,1\right)\right)\right)=\left(A, \oplus, \boxplus,^{-}, \sim, 0,1\right)$.

(2) Let $(A, \wedge, \vee, *, \rightsquigarrow, \rightarrow, 0,1)$ be a pseudo residuated l-groupoid that satisfies the conditions of Proposition 3.14, then

$$
H(G((A, \wedge, \vee, *, \rightsquigarrow, \rightarrow, 0,1)))=(A, \wedge, \vee, *, \rightsquigarrow, \rightarrow, 0,1) .
$$

Proof. (1) By Proposition 3.6, $H\left(\left(A, \oplus, \boxplus,^{-}, \sim, 0,1\right)\right):=(A, \wedge, \vee, \odot, \rightsquigarrow, \rightarrow, 0,1)$ is a pseudo residuated $l$-groupoid. Using Proposition 3.2 part (3) and part (4), we have

(i)' $\left(y^{\sim} \rightarrow x^{\sim}\right) \odot x=(x \rightsquigarrow y) \odot x=x \wedge y$;

(ii) $)^{\prime} x \odot\left(y^{\sim} \rightarrow x^{\sim}\right)=(x \rightsquigarrow y) \triangleright x=x \wedge y$. 


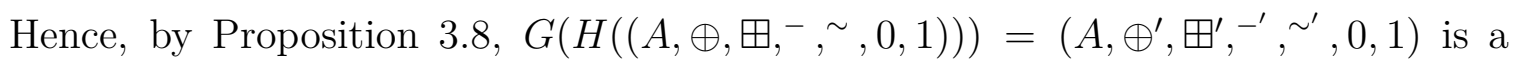
pseudo commutative double basic algebra where $x^{{{ }^{\prime}}^{\prime}}=x \rightarrow 0, x^{\sim^{\prime}}=x \rightsquigarrow 0, x \oplus^{\prime} y=$

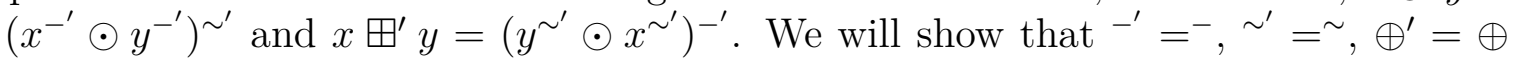
and $\boxplus^{\prime}=\boxplus$. It is obvious that ${ }^{{ }^{\prime}}={ }^{-}$and ${ }^{\sim^{\prime}}=\sim$. We have

$$
\begin{aligned}
& x \oplus^{\prime} y=\left(x^{-^{\prime}} \odot y^{-^{\prime}}\right)^{\sim^{\prime}}=\left(x^{-} \odot y^{-}\right)^{\sim}=x \oplus y, \\
& x \boxplus^{\prime} y=\left(y^{\sim^{\prime}} \odot x^{\sim^{\prime}}\right)^{-^{\prime}}=\left(y^{\sim} \odot x^{\sim}\right)^{-}=\left(x^{\sim} \boxminus y^{\sim}\right)^{-}=x \boxplus y .
\end{aligned}
$$

Hence, $G\left(H\left(\left(A, \oplus, \boxplus,^{-}, \sim, 0,1\right)\right)\right)=\left(A, \oplus, \boxplus,^{-}, \sim, 0,1\right)$.

(2) $G((A, \wedge, \vee, *, \rightsquigarrow, \rightarrow, 0,1)):=\left(A, \oplus, \boxplus,^{-}, \sim, 0,1\right)$ is a pseudo commutative double basic algebra like Proposition 3.7. Hence, $H(G((A, \wedge, \vee, *, \rightarrow, \rightsquigarrow, 0,1)))$ is a pseudo residuated $l$-groupoid satisfying conditions of Proposition 3.7. Suppose that

$$
H(G((A, \wedge, \vee, *, \rightarrow, \rightsquigarrow, 0,1)))=\left(A, \wedge^{\prime}, \vee^{\prime}, *^{\prime}, \rightsquigarrow^{\prime}, \rightarrow^{\prime}, 0,1\right) .
$$

We have

$$
\begin{aligned}
x *^{\prime} y & =x \odot y=\left(x^{\sim} \oplus y^{\sim}\right)^{-}=\left(x^{-\sim} * y^{-\sim}\right)^{\sim-}=x * y, \\
x \rightarrow^{\prime} y & =x^{\sim} \oplus y=\left(x * y^{-}\right)^{\sim}=x \rightsquigarrow y, \\
x \aleph^{\prime} y & =x^{-} \boxplus y=\left(y^{\sim} * x\right)^{-}=x \rightarrow y, \\
x \wedge^{\prime} y & =x *^{\prime}\left(y^{\sim^{\prime}} \rightarrow x^{\sim^{\prime}}\right)=\left(y^{-} \rightsquigarrow x^{-}\right) * x=x \wedge y, \\
x \vee^{\prime} y & =\left(x^{-^{\prime}} \wedge^{\prime} y^{-^{\prime}}\right)^{\sim^{\prime}}=\left(x^{\sim} \wedge y^{\sim}\right)^{-}=x \vee y .
\end{aligned}
$$

Proposition 3.9. Let $\left(A, \oplus, \boxplus,^{-},^{\sim}, 0,1\right)$ be a double $M V$-algebra. Then $(A, \wedge, \vee, \odot, \rightsquigarrow, \rightarrow, 1)$ and $(A, \wedge, \vee, \odot, \rightarrow, \rightsquigarrow, 1)$ are pseudo hoops.

Proof. Let $\left(A, \oplus, \boxplus,^{-}, \sim, 0,1\right)$ be a double MV-algebra. Then $\left(A, \oplus, \boxplus,^{-}, \sim, 0,1\right)$ is a pseudo commutative double basic algebra. Hence, $(A, \wedge, \vee, \square, \rightarrow, \rightsquigarrow, 0,1)$ is a pseudo residuated $l$-groupoid by Proposition 3.6. Since $\boxplus$ is associative, then $\square$ is associative. By Proposition 3.2 part $(8)(((x \boxminus y) \rightarrow z) \square x) \square y=((x \boxminus y) \rightarrow z) \square(x \boxminus y) \leq z$ and $(((x \boxminus y) \rightsquigarrow z) \odot y) \odot x=((x \boxminus y) \rightsquigarrow z) \odot(y \odot x)=((x \boxminus y) \rightsquigarrow z) \odot(x \boxminus y) \leq z$. So, $(x \boxminus y) \rightarrow z \leq x \rightarrow(y \rightarrow z)$ and $(x \boxminus y) \rightsquigarrow z \leq y \rightsquigarrow(x \rightsquigarrow z)$ by Proposition 3.2 part (9) and part (10). On the other hand

$$
\begin{aligned}
& (x \rightarrow(y \rightarrow z)) \bullet(x \boxminus y)=((x \rightarrow(y \rightarrow z)) \bullet x) \bullet y \leq(y \rightarrow z) \bullet y \leq z, \\
& (y \rightsquigarrow(x \rightsquigarrow z)) \odot(x \boxminus y)=(((y \rightsquigarrow(x \rightsquigarrow z)) \odot y) \odot x) \leq(x \rightsquigarrow z) \odot x \leq z .
\end{aligned}
$$

Hence, $(A, \wedge, \vee, \odot, \rightsquigarrow, \rightarrow, 1)$ is a pseudo hoop. Similarly, we can prove $(x \odot y) \rightsquigarrow z=$ $x \rightsquigarrow(y \rightsquigarrow z)$ and $(x \odot y) \rightarrow z=y \rightarrow(x \rightarrow z)$. Hence, $(A, \wedge, \vee, \odot, \rightsquigarrow, \rightarrow, 1)$ is a pseudo hoop.

Corollary 3.5. Let $\left(A, \oplus, \boxplus,^{-}, \sim, 0,1\right)$ be a double $M V$-algebra. Then $(A, \leq, \rightarrow, \rightsquigarrow, 1)$ is a pseudo BCK-algebra.

Proof. We know that every pseudo-hoop is a pseudo-BCK algebra which is a meet semilattice satisfying the pseudo-divisibility property. Since $(A, \wedge, \vee, \odot, \rightsquigarrow, \rightarrow, 1)$ is a pseudo hoop by Proposition 3.9 , then $(A, \leq, \rightarrow, \rightsquigarrow, 1)$ is a pseudo BCK-algebra. 


\section{Boolean Center of Pseudo Commutative Double Basic Algebras}

Let $(L, \wedge, \vee, 0,1)$ be a bounded lattice. Recall that (see [1,21]) an element $a \in L$ is said to be complemented if there is an element $b \in L$ such that $a \vee b=1$ and $a \wedge b=0$. If such an element $b$ exists, then it is called a complement of $a$. Complements are generally not unique unless the lattice is distributive. Hence in pseudo commutative double basic algebras the complements are unique.

Proposition 4.1. Let $\left(A, \oplus, \boxplus,^{-}, \sim, 0,1\right)$ be a pseudo commutative double basic algebra and $a \in A$ an element which has a complement $b \in A$. Then $a^{-}=a^{\sim}=b$ and $b^{-}=b^{\sim}=a$.

Proof. We have $1=a \vee b \leq a \boxplus b$. Hence, $a \boxplus b=1$. Thus, $a^{\sim} \leq b$. On the other hand, we have that $a^{\sim}=0 \boxplus a^{\sim}=(b \wedge a) \boxplus a^{\sim}=\left(b \boxplus a^{\sim}\right) \wedge\left(a \boxplus a^{\sim}\right)=$ $\left(b \boxplus a^{\sim}\right) \wedge\left(a^{\sim} \oplus a\right)=\left(b \boxplus a^{\sim}\right) \wedge 1=\left(b \boxplus a^{\sim}\right)$ by Proposition 2.4 part (12) and part (3). Since $\left(b \vee a^{\sim}\right) \leq\left(b \boxplus a^{\sim}\right)=a^{\sim}$, then $b \leq a^{\sim}$. Therefore, $a^{\sim}=b$ and $a=a^{\sim-}=b^{-}$. Similarly, we can prove $a^{-}=b$ and $b^{\sim}=a$.

Let $\left(A, \oplus, \boxplus,^{-}, \sim, 0,1\right)$ be a pseudo commutative double basic algebra. We denote by $B(A)$ the boolean algebra associated with the bounded distributive lattice $L(A)=$ $(A, \wedge, \vee, 0,1)$. The set $B(A)$ is called the boolean center of $A$ and elements of $\mathrm{B}(\mathrm{A})$ are called the boolean elements of $A$.

Proposition 4.2. Let $\left(A, \oplus, \boxplus,^{-}, \sim, 0,1\right)$ be a pseudo commutative double basic algebra. Then the following are equivalent:
(1) $a \in B(A)$;
(2) $a \wedge a^{-}=0$
(3) $a \oplus a=a$;
(4) $a \boxplus a=a$;
(5) $a \wedge a^{\sim}=0$;
(6) $a \vee a^{-}=1$;
(7) $a \odot a=a$;
(8) $a \boxminus a=a$;
(9) $a \vee a^{\sim}=1$.

Proof. (1) $\Rightarrow(2)$ Since $a \in B(A)$, then $a$ has a complement $b \in A$. By Proposition 4.1, we have $b=a^{-}$. Hence, $a \wedge a^{-}=0$.

$(2) \Rightarrow(3)$ By Proposition 2.2 part (12) and part (3), we obtain $a=0 \oplus a=(a \wedge$ $\left.a^{\sim}\right) \oplus a=(a \oplus a) \wedge\left(a^{\sim} \oplus a\right)=a \oplus a$.

$(3) \Rightarrow(4)$ Since $A$ is pseudo commutative, it is clear.

(4) $\Rightarrow(5)$ Let $a \boxplus a=a$. By Proposition 2.2 part (3), we have

$$
a \wedge a^{\sim}=\left(a^{-} \vee a\right)^{\sim}=\left((a \boxplus a)^{\sim} \oplus a\right)^{-}=\left(a^{\sim} \oplus a\right)^{-}=1^{-}=0 .
$$

$(5) \Rightarrow(6)$ We have $0=a \wedge a^{\sim}=\left(a^{-} \vee a^{\sim-}\right)^{\sim}=\left(a^{-} \vee a\right)^{\sim}$. Hence $a^{-} \vee a=\left(a \wedge a^{\sim}\right)^{-}=$ $0^{-}=1$. 
$(6) \Rightarrow(7)$ Using Proposition 3.1 part (5) and Proposition 3.2 part (7), we get $a=$ $1 \odot a=\left(a \vee a^{-}\right) \odot a=(a \odot a) \vee\left(a \odot a^{-}\right)=(a \odot a) \vee 0=a \odot a$.

$(7) \Rightarrow(8)$ It is clear.

(8) $\Rightarrow(9)$ Suppose $a \square a=a$. Then $a^{-} \boxplus a^{-}=a^{-}$. Similar to (4) $\Rightarrow(5)$, we can prove $a^{-} \wedge a^{-\sim}=0$. Hence, $a \vee a^{\sim}=1$.

$(9) \Rightarrow(1)$ Since $a \vee a^{\sim}=1$, then $a \wedge a^{-}=0$. Similar to $(2) \Rightarrow(3)$, we can show that $a \oplus a=a$. Thus, $a \boxplus a=a$. Then, similar to (4) $\Rightarrow(5)$, we obtain $a \wedge a^{\sim}=0$.

Proposition 4.3. Let $\left(A, \oplus, \boxplus,^{-}, \sim, 0,1\right)$ be a pseudo commutative double basic algebra and $a \in B(A)$. Then the following hold:

(1) $a^{-}=a \rightarrow a^{-}=a \rightsquigarrow a^{-}=a \rightarrow a^{\sim}=a \rightsquigarrow a^{\sim}=a^{\sim}$;

(2) $a^{-} \rightarrow a=a^{-} \rightsquigarrow a=a^{\sim} \rightarrow a=a^{\sim} \rightsquigarrow a=a$.

Proof. (1) We have $1=a \vee a^{-}=\left(a \rightsquigarrow a^{-}\right) \rightarrow a^{-}=\left(a \rightarrow a^{-}\right) \rightsquigarrow a^{-}$by (C4). Hence, $a \rightsquigarrow a^{-} \leq a^{-}$and $a \rightarrow a^{-} \leq a^{-}$. Since $a^{-} \leq a \rightsquigarrow a^{-}$and $a^{-} \leq a \rightarrow a^{-}$, then $a \rightarrow a^{-}=a \rightsquigarrow a^{-}=a^{-}$. By Proposition 3.2 part (4), we have $a \rightarrow a^{\sim}=a \rightsquigarrow a^{-}=a^{-}$. Similarly, we can prove $a \rightarrow a^{\sim}=a \rightsquigarrow a^{\sim}=a^{\sim}$.

(2) It follows from (1) and Proposition 3.2 part (3).

Proposition 4.4. Let $\left(A, \oplus, \boxplus,^{-}, \sim, 0,1\right)$ be a pseudo commutative double basic algebra, $a \in B(A)$ and $x, y \in A$. Then the following hold:

(1) $a \oplus x=x \oplus a=a \vee x=a \boxplus x=x \boxplus a ;$

(2) $a \odot x=x \odot a=a \wedge x=a \boxminus x=x \boxminus a$;

(3) $a \vee(x \odot y)=(a \vee x) \odot(a \vee y), a \vee(x \boxminus y)=(a \vee x) \odot(a \vee y)$;

(4) $a \wedge(x \oplus y)=(a \wedge x) \oplus(a \wedge y), a \wedge(x \boxplus y)=(a \wedge x) \boxplus(a \wedge y)$.

Proof. (1) Since $A$ is pseudo commutative, we have $a \vee x \leq a \oplus x$. Using Proposition 3.2 part $(13)$ and $(\mathrm{C} 6)$, we get $(a \oplus x) \odot(a \vee x)^{\sim}=(a \oplus x) \odot\left(a^{\sim} \wedge x^{\sim}\right)=\left((a \oplus x) \odot a^{\sim}\right) \wedge$ $\left((a \oplus x) \odot x^{\sim}\right)=\left((a \oplus x) \odot a^{\sim}\right) \wedge\left(a \wedge x^{\sim}\right) \leq\left(a^{-} \wedge a\right)=0$. Hence $(a \oplus x)^{-} \boxplus(a \vee x)=1$ implies that $a \oplus x \leq a \vee x$. Since $A$ is pseudo commutative, then $a \oplus x=a \vee x=x \oplus a$. Similarly, we can prove $a \vee x=a \boxplus x=x \boxplus a$.

(2) It follows from part (1) and the definitions of $\odot$ and $\square$.

(3) By Proposition 3.2 part (7), Proposition 4.2 part (7) and Proposition 3.1 part (6), we have $(a \vee x) \odot(a \vee y)=(a \odot(a \vee y)) \vee(x \odot(a \vee y))=(a \odot a) \vee(a \odot y) \vee(x \odot a) \vee(x \odot y)=$ $a \vee(x \odot y)$.

(4) Using Proposition 2.2 part (12) Proposition 4.2 part (3) and Proposition 2.2 part (6), we obtain

$$
\begin{aligned}
(a \wedge x) \oplus(a \wedge y) & =(a \oplus(a \wedge y)) \vee(x \oplus(a \wedge y)) \\
& =(a \oplus a) \wedge(a \oplus y) \wedge(x \oplus a) \wedge(x \oplus y) \\
& =a \wedge(x \oplus y)
\end{aligned}
$$

Proposition 4.5. Let $\left(A, \oplus, \boxplus,^{-}, \sim, 0,1\right)$ be a pseudo commutative double basic algebra. Then $B(A)$ is a boolean subalgebra of $A$. 
Proof. Clearly $0,1 \in B(A)$. Let $a, b \in B(A)$. By Proposition 4.1, we have $a \wedge a^{-}=0$, $a \vee a^{-}=1, b \wedge b^{-}=0, b \vee b^{-}=1$. We will prove that $a^{-} \vee b^{-}$is the complement of $a \wedge b$ and

$$
\begin{aligned}
& (a \wedge b) \wedge\left(a^{-} \vee b^{-}\right)=\left((a \wedge b) \wedge a^{-}\right) \vee\left((a \wedge b) \wedge b^{-}\right)=0 \vee 0=0, \\
& (a \wedge b) \vee\left(a^{-} \vee b^{-}\right)=\left(a \vee\left(a^{-} \vee b^{-}\right)\right) \wedge\left(b \vee\left(a^{-} \vee b^{-}\right)\right)=1 \wedge 1=1 .
\end{aligned}
$$

Similarly, we can show that $a^{-} \vee b^{-}$is the complement of a $a \wedge b$. Hence $a \wedge b, a \vee b \in B(A)$ and $(B(A), \wedge, \vee)$ is a distributive lattice. By Proposition 4.4 part (1), we have $a \oplus b=a \vee b \in B(A)$ and $a \boxplus b=a \vee b \in B(A)$. It is clear that $a^{-}, a^{\sim} \in B(A)$. Hence $B(A)$ is closed under the operations $\oplus, \boxplus,{ }^{-}$and $\sim$.

Corollary 4.1. Let $\left(A, \oplus, \boxplus,^{-}, \sim, 0,1\right)$ be a pseudo commutative double basic algebra. Then $\left(B(A), \wedge, \vee,^{-}\right)$is a De Morgan lattice.

Proposition 4.6. Let $\left(A, \oplus, \boxplus,^{-}, \sim, 0,1\right)$ be a pseudo commutative double basic algebra and $a \in B(A)$. Then $\left([0, a], \oplus_{0}^{a}, \boxplus_{0}^{a},{ }_{0}^{a}, \sim_{0}^{a}, 0, a\right)$ is a pseudo commutative double basic algebra, where

$$
\begin{aligned}
x^{-a}: & :=x^{-} \odot a, \quad x^{\sim_{0}^{a}}:=x^{\sim} \boxminus a, \\
x \oplus_{0}^{a} y & :=x \oplus y, \quad x \boxplus_{0}^{a} y:=x \boxplus y .
\end{aligned}
$$

Proof. Let $x, y \in[0, a]$ be arbitrary. Using pseudo commutativity, Proposition 2.2 part (5) and Proposition 4.2 part (3) and part (4), we have $0 \leq x \oplus y \leq a \oplus a=a$, $0 \leq x \boxplus y \leq a \boxplus a=a$. Thus $x \oplus y, x \boxplus y \in[0, a]$. By Proposition 3.1 part (6), we obtain $x^{-a}:=x^{-} \odot a \leq a$ and $x^{\sim_{0}^{a}}:=x^{\sim} \square a \leq a$. So, $x^{-{ }_{0}^{a}}, x^{\sim_{0}^{a}} \in[0, a]$. We will check condition (P2) from the definition of a double basic algebra.

Applying Corollary 3.1, Proposition 4.4 part (1), Proposition 3.2 part (7) and Proposition 4.2, we get

$$
\begin{aligned}
\left(x^{-a}\right)^{\sim} \sim_{0}^{a} & =\left(x^{-} \odot a\right)^{\sim} \triangleright a=\left(x \oplus a^{\sim}\right) \triangleright a=a \odot\left(x \vee a^{\sim}\right)=(a \odot x) \vee\left(a \odot a^{\sim}\right) \\
& =(a \wedge x) \vee\left(a \wedge a^{\sim}\right)=x \vee 0=x, \\
\left(x^{\sim_{0}^{a}}\right)^{-a} & =\left(x^{\sim} \bullet a\right)^{-} \odot a=\left(x \boxplus a^{-}\right) \odot a=a \boxminus\left(x \vee a^{-}\right)=(a \boxminus x) \vee\left(a \boxminus a^{-}\right) \\
& =(a \wedge x) \vee\left(a \wedge a^{-}\right)=x \vee 0=x .
\end{aligned}
$$

It is easy to prove the other identities.

Proposition 4.7. Let $\left(A, \oplus, \boxplus,^{-}, \sim, 0,1\right)$ be a pseudo commutative double basic algebra with $a, b \in B(A)$ and $a \leq b$. Then $\left([a, b], \oplus_{a}^{b}, \boxplus_{a}^{b},{ }^{-b}, \sim_{a}^{b}, a, b\right)$ is a pseudo commutative double basic algebra, where

$$
\begin{aligned}
x^{-b} a & :=\left(x^{-} \odot b\right) \boxplus a, \quad x^{\sim_{a}^{b}}:=\left(x^{\sim} \boxminus b\right) \oplus a, \\
x \oplus_{a}^{b} y & :=\left(\left(x^{-} \boxplus a\right)^{\sim} \oplus y\right) \wedge b, \quad x \boxplus_{a}^{b} y:=\left(\left(x^{\sim} \oplus a\right)^{-} \boxplus y\right) \wedge b .
\end{aligned}
$$

Proof. It could be easily proven by Proposition 4.6 and Theorem 4 in [12]. 
Acknowledgements The author would like to express her thanks to referees for their comments and suggestions which improved the paper.

\section{REFERENCES}

[1] R. Balbes and P. Dwinger, Distributive Lattices, University of Missouri Press, Columbia, 1974.

[2] B. Bosbach, Komplementare Halbgruppen. Axiomatik und aritmetik, Fundam. Math. 64 (1969), 257-287.

[3] B. Bosbach, Komplementare Halbgruppen. Kongruenzen und quotienten, Fundam. Math. 69 (1970), 1-14.

[4] B. Bosbach, Residuation groupoids, Results Math. 5 (1982), 107-122.

[5] M. Botur and R. Halas, Finite commutative basic algebras are MV-algebras, J. Mult.-Valued Logic Soft Comput. 14 (2008), 69-80.

[6] M. Botur and R. Halas, Commutative basic algebras and non-associative fuzzy logics, Arch. Math. Logic 48 (2009), 243-255.

[7] M. Botur, I. Chajda and R. Halas, Are basic algebras residuated structures? Soft Comput. 14 (2010), 251-255.

[8] I. Chajda, R. Halas and J. Kuhr, Distributive lattices with sectionally antitone involutions, Acta Sci. Math. (Szeged) 71 (2005), 19-33.

[9] I. Chajda and J. Kuhr, A note on interval MV-algebras, Math. Slovaca 56 (2006), 47-52.

[10] I. Chajda, R. Halas and J. Kuhr, Semilattice Structures, Research and Exposition in Mathematics 30, Heldermann, Verlag, 2007.

[11] I. Chajda, R. Halas and J. Kuhr, Many-valued quantum algebras, Algebra Universalis 60 (2009), 63-90.

[12] I. Chajda, Double basic algebras, Order 26 (2009), 149-162.

[13] I. Chajda, M. Kolarik and J. Kuhr, On double basic algebras and pseudo-effect algebras, Order 28 (2011), 499-512.

[14] I. Chajda, M. Kolarik and J. Krnavek, Pseudo Basic Algebras, J. Mult.-Valued Logic Soft Comput. 21 (2013), 113-129.

[15] I. Chajda, Basic algebras, logics, trends and applications, Asian-Eur. J. Math. 8 (2015), Paper ID 1550040, 46 pages.

[16] C. C. Chang, Algebraic analysis of many valued logics, Trans. Amer. Math. Soc. 88 (1958), 464-490.

[17] L. Ciungu, Non-commutative Multiple-Valued Logic Algebras, Springer International Publishing Switzerland, Basel, 2014.

[18] A. Dvurečenskij and S. Pulmannová, New Trends in Quantum Structures, Kluwer, Dordrecht, 2000.

[19] G. Georgescu and A. Iorgulescu, Pseudo-BCK algebras: an extension of BCK-algebras, In: Proc. DMTCSS01: Combinatorics, Computability and Logic, Springer, London, 2001, 97-114.

[20] Sh. Ghorbani, Localization of hoop-algebras, J. Adv. Res. Pure Math. 5 (2013), 1-13.

[21] G. Gratzer, Lattice Theory. First Concepts and Distributive Lattices, A Series of Books in Mathematics, Freeman, San Francisco, 1972.

[22] P. Hájek, Metamathematics of Fuzzy Logic, Kluwer, Dordrecht, 1998.

[23] M. Ward and R. P. Dilworth, Residuated lattices, Trans. Amer. Math. Soc. 45 (1939), 335-354. 
${ }^{1}$ Department of Pure Mathematics,

FACUlty OF MATHEMATiCs AND COMPUTER,

Shahid BAHONAR University OF KERMAN,

KERMAN, IRAN

Email address: sh.ghorbani@uk.ac.ir 